\title{
THE EQUIVALENCE BETWEEN THE CONVERGENCES \\ OF MANN AND ISHIKAWA ITERATION METHODS WITH \\ ERRORS FOR DEMICONTINUOUS $\phi$-STRONGLY ACCRETIVE \\ OPERATORS IN UNIFORMLY SMOOTH BANACH SPACES
}

\author{
ZEQING LIU, LI WANG, JEONG SHEOK UME, AND SHIN MIN KANG
}

Received 22 October 2004; Revised 5 March 2006; Accepted 12 March 2006

We investigate the equivalence between the convergences of the Mann iteration method and the Ishikawa iteration method with errors for demicontinuous $\phi$-strongly accretive operators in uniformly smooth Banach spaces. A related result deals with the equivalence of the Mann iteration method and the Ishikawa iteration method for $\phi$-pseudocontractive operators in nonempty closed convex subsets of uniformly smooth Banach spaces. The results presented in this paper extend and improve the corresponding results in the literature.

Copyright @ 2006 Hindawi Publishing Corporation. All rights reserved.

\section{Introduction and preliminaries}

For a Banach space $X$ we will denote by $J$ the normalized duality mapping from $X$ into $2^{X^{*}}$ given by

$$
J(x)=\left\{f^{*} \in X^{*}: \operatorname{Re}\left\langle x, f^{*}\right\rangle=\left\|f^{*}\right\|^{2}=\|x\|^{2}\right\}, \quad x \in X,
$$

where $X^{*}$ denotes the dual space of $X$ and $\langle\cdot, \cdot\rangle$ denotes the generalized duality pairing. It is known that $X$ is a uniformly smooth Banach space if and only if $J$ is single-valued and uniformly continuous on any bounded subset of $X$. Let $I$ denote the identity operator in $X$.

An operator $T$ with domain $D(T)$ and range $R(T)$ in $X$ is said to be strongly accretive if there exists a constant $k>0$ such that for any $x, y \in D(T)$, there exists $j(x-y) \in J(x-y)$ satisfying

$$
\operatorname{Re}\langle T x-T y, j(x-y)\rangle \geq k\|x-y\|^{2} .
$$

Without loss of generality we may assume that $k \in(0,1)$. It is known that $T$ is accretive if and only if for any $x, y \in D(T)$, there exists $j(x-y) \in J(x-y)$ such that

$$
\operatorname{Re}\langle T x-T y, j(x-y)\rangle \geq 0 .
$$

Hindawi Publishing Corporation International Journal of Mathematics and Mathematical Sciences Volume 2006, Article ID 69542, Pages 1-12

DOI 10.1155/IJMMS/2006/69542 
Furthermore, $T$ is called $\phi$-strongly accretive if there exists a strictly increasing function $\phi:[0, \infty) \rightarrow[0, \infty)$ with $\phi(0)=0$ such that for any $x, y \in D(T)$ there exists $j(x-y) \in$ $J(x-y)$ satisfying

$$
\operatorname{Re}\langle T x-T y, j(x-y)\rangle \geq \phi(\|x-y\|)\|x-y\| .
$$

Closely related to the class of strongly accretive operators is the class of strongly pseudocontractive operators where an operator $T$ is called strongly pseudocontractive if there exists $t>1$ such that for any $x, y \in D(T)$, there exists $j(x-y) \in J(x-y)$ satisfying

$$
\operatorname{Re}\langle T x-T y, j(x-y)\rangle \leq \frac{1}{t}\|x-y\|^{2} .
$$

$T$ is called $\phi$-strongly pseudocontractive if there exists a strictly increasing function $\phi$ : $[0, \infty] \rightarrow[0, \infty]$ with $\phi(0)=0$ such that for any $x, y \in D(T)$ there exists $j(x-y) \in$ $J(x-y)$ satisfying

$$
\operatorname{Re}\langle T x-T y, j(x-y)\rangle \leq\|x-y\|^{2}-\phi(\|x-y\|)\|x-y\| .
$$

An operator $A: X \rightarrow X^{*}$ is said to be demicontinuous on $X$ if $\left\{A x_{n}\right\}_{n \geq 1}$ converges weakly to $A x_{0}$ for any $x_{0} \in X$ and $\left\{x_{n}\right\}_{n \geq 1} \subset X$ with $\lim _{n \rightarrow \infty} x_{n}=x_{0}$. It is well known that if $X$ is a finite-dimensional space, then $A$ is demicontinuous if and only if it is continuous.

Within the past 20 years or so, various authors have applied the Mann iteration method, the Mann iteration method with errors, the Ishikawa iteration method, and the Ishikawa iteration method with errors to approximate fixed points of pseudocontractive, strongly pseudocontractive, $\phi$-strongly pseudocontractive, and to approximate solutions of nonlinear equations $T x=f$ and $x+T x=f$ in the case when $T$ is accretive, strongly accretive, and $\phi$-strongly accretive (see, e.g., $[1,3-15,20]$ and the references therein). Recently, the equivalence of the Mann iteration method and the Ishikawa iteration method for various nonlinear operators and nonlinear equations has been established in Banach spaces or uniformly smooth Banach spaces. For details, we refer to [2, 16-19]. Especially, Rhoades and Soltuz [18] obtained the equivalence of the Mann iteration method and the Ishikawa iteration method for strongly pseudocontractive operators, strongly accretive operators, and accretive operators, respectively, in uniformly smooth Banach spaces.

It is our purpose in this paper to show the equivalence of both the Mann iteration method with errors and the Ishikawa iteration method with errors for $\phi$-strongly accretive operators in uniformly smooth Banach spaces, and the Mann iteration method and the Ishikawa iteration method for $\phi$-strongly pseudocontractive operators in nonempty closed and convex subsets of uniformly smooth Banach spaces. The results presented in this paper extend, improve, and unify the corresponding results due to Chang [1], Chang et al. cite3, Chidume [4-6], Chidume and Osilike [7], Osilike [14], Rhoades and Soltuz 
[18], Zhou [20], and others. Two examples which dwell upon the importance of our results are given.

The following lemmas play crucial roles in the proofs of our main results.

Lemma 1.1 [11]. Suppose that $X$ is a uniformly smooth Banach space and $T: X \rightarrow X$ is a demicontinuous $\phi$-strongly accretive operator. Then the equation $T x=f$ has a unique solution for any $f \in X$.

Lemma 1.2 [15]. Let $X$ be a uniformly smooth Banach space. Then there exists a nondecreasing continuous function $b:[0, \infty) \rightarrow[0, \infty)$ satisfying the conditions:

(i) $b(0)=0, b(c t) \leq c b(t)$ for any $t \geq 0$ and $c \geq 1$, and

(ii) $\|x+y\|^{2} \leq\|x\|^{2}+2 \operatorname{Re}\langle y, J(x)\rangle+\max \{\|x\|, 1\}\|y\| b(\|y\|)$ for any $x, y \in X$.

\section{Main results}

In the following we establish the equivalence between the convergence of the Mann iteration method with errors and the Ishikawa iteration method with errors for demicontinuous strongly accretive operators in uniformly smooth Banach spaces.

Theorem 2.1. Let $X$ be a uniformly smooth Banach space and $T: X \rightarrow X$ a demicontinuous $\phi$-strongly accretive operator. For a given $f \in X$, let $S x=f+x-T x$ for any $x \in X$. Define the Ishikawa iteration sequence with errors $\left\{x_{n}\right\}_{n \geq 0}$ iteratively by

$$
\begin{gathered}
x_{0}, \sigma_{0}, \delta_{0} \in X, \\
y_{n}=\left(1-b_{n}\right) x_{n}+b_{n} S x_{n}+\delta_{n}, \\
x_{n+1}=\left(1-a_{n}\right) x_{n}+a_{n} S y_{n}+\sigma_{n}, \quad n \geq 0,
\end{gathered}
$$

and the Mann iteration sequence with errors $\left\{u_{n}\right\}_{n \geq 0}$ iteratively by

$$
\begin{gathered}
x_{0}, \sigma_{0} \in X, \\
u_{n+1}=\left(1-a_{n}\right) u_{n}+a_{n} S u_{n}+\delta_{n}, \quad n \geq 0,
\end{gathered}
$$

where $\left\{\sigma_{n}\right\}_{n \geq 0},\left\{\delta_{n}\right\}_{n \geq 0}$ are sequences in $X$ and $\left\{a_{n}\right\}_{n \geq 0},\left\{b_{n}\right\}_{n \geq 0}$ are sequences in $[0,1]$ satisfying

$$
\begin{gathered}
\sum_{n=0}^{\infty} a_{n}=\infty, \quad \sum_{n=0}^{\infty}\left\|\sigma_{n}\right\|<\infty, \\
\lim _{n \rightarrow \infty} a_{n}=\lim _{n \rightarrow \infty} b_{n}=\lim _{n \rightarrow \infty}\left\|\delta_{n}\right\|=0 .
\end{gathered}
$$

Assume that

$$
\begin{aligned}
& \text { either the sequences }\left\{x_{n}-T x_{n}\right\}_{n \geq 0} \text { and }\left\{y_{n}-T y_{n}\right\}_{n \geq 0} \\
& \text { or the sequences }\left\{T x_{n}\right\}_{n \geq 0} \text { and }\left\{T y_{n}\right\}_{n \geq 0} \text { are bounded. }
\end{aligned}
$$

Then, for $u_{0}=x_{0} \in X$, the following assertions are equivalent:

(i) the Mann iteration sequence with errors $\left\{u_{n}\right\}_{n \geq 0}$ converges strongly to the unique solution of the equation $T x=f$; 
4 The equivalence of Mann and Ishikawa iteration methods

(ii) the Ishikawa iteration sequence with errors $\left\{x_{n}\right\}_{n \geq 0}$ converges strongly to the unique solution of the equation $T x=f$.

Proof. It follows from Lemma 1.1 that the equation $T x=f$ has a unique solution $q \in X$. It is clear that $S$ is demicontinuous and $q$ is a unique fixed point of $S$. Thus (i) follows from (ii) by setting $b_{n}=0$ and $\delta_{n}=0$ for any $n \geq 0$.

Next we prove that (i) implies (ii). Since $T$ is $\phi$-strongly accretive, it follows that for any $x, y \in X$

$$
\operatorname{Re}\langle S x-S y, J(x-y)\rangle \leq\|x-y\|^{2}(1-A(\|x-y\|))
$$

where $A(\|x-y\|)=\phi(\|x-y\|) /(1+\|x-y\|+\phi(\|x-y\|)) \in[0,1)$ for all $x, y \in X$. Set

$$
\begin{gathered}
d=\sup \left\{\left\|S x_{n}-q\right\|+\left\|S y_{n}-q\right\|+\left\|S u_{n}-q\right\|: n \geq 0\right\}+\left\|x_{0}-q\right\|+\left\|u_{0}-q\right\|, \\
N=\sup \left\{\left\|\delta_{n}\right\|: n \geq 0\right\}, \quad M=d+\sum_{n=0}^{\infty}\left\|\sigma_{n}\right\|, \quad L=M+N .
\end{gathered}
$$

It follows from the $\phi$-strong accretivicity of $T$ that for any $x, y \in X$,

$$
\operatorname{Re}\langle T x-T y, J(x-y)\rangle \geq \phi(\|x-y\|)\|x-y\|,
$$

which implies that

$$
\phi(\|x-y\|) \leq\|T x-T y\| \quad \forall x, y \in X
$$

Observe that

$$
\begin{gathered}
\|S x-S y\| \leq\|x-y\|+\|T x-T y\| \leq \phi^{-1}(\|T x-T y\|)+\|T x-T y\|, \\
\|S x-S y\| \leq\|x-T x\|+\|y-T y\|
\end{gathered}
$$

for all $x, y \in X$. Therefore, $d, M$, and $L$ are bounded by (2.3)-(2.5). It is evident to verify that

$$
\begin{gathered}
\max \left\{\left\|u_{n}-q\right\|,\left\|x_{n}-q\right\|\right\} \leq d+\sum_{k=0}^{n}\left\|\sigma_{k}\right\| \leq M, \\
\left\|y_{n}-q\right\| \leq\left(1-b_{n}\right)\left\|x_{n}-q\right\|+b_{n}\left\|S x_{n}-q\right\|+\left\|\delta_{n}\right\| \leq L
\end{gathered}
$$


for any $n \geq 0$. In terms of Lemma 1.2, (2.1), and (2.2), we arrive at

$$
\begin{aligned}
\left\|y_{n}-u_{n}\right\|^{2}= & \left\|\left(1-b_{n}\right)\left(x_{n}-u_{n}\right)+b_{n}\left(S x_{n}-u_{n}\right)+\delta_{n}\right\|^{2} \\
\leq & \left\|\left(1-b_{n}\right)\left(x_{n}-u_{n}\right)+b_{n}\left(S x_{n}-u_{n}\right)\right\|^{2} \\
& +2 \operatorname{Re}\left\langle\delta_{n}, J\left(\left(1-b_{n}\right)\left(x_{n}-u_{n}\right)+b_{n}\left(S x_{n}-u_{n}\right)\right)\right\rangle \\
& +\max \left\{\left\|\left(1-b_{n}\right)\left(x_{n}-u_{n}\right)+b_{n}\left(S x_{n}-u_{n}\right)\right\|, 1\right\}\left\|\delta_{n}\right\| b\left(\left\|\delta_{n}\right\|\right) \\
\leq & \left(1-b_{n}\right)^{2}\left\|x_{n}-u_{n}\right\|^{2}+2 b_{n}\left(1-b_{n}\right) \operatorname{Re}\left\langle S x_{n}-u_{n}, J\left(x_{n}-u_{n}\right)\right\rangle \\
& +\max \left\{\left\|\left(1-b_{n}\right)\left(x_{n}-u_{n}\right)\right\|, 1\right\} b_{n}\left\|S x_{n}-u_{n}\right\| b\left(b_{n}\left\|S x_{n}-u_{n}\right\|\right) \\
& +D_{1}\left\|\delta_{n}\right\|+D_{2}\left\|\delta_{n}\right\| b\left(\left\|\delta_{n}\right\|\right) \\
\leq & \left(1-b_{n}\right)^{2}\left\|x_{n}-u_{n}\right\|^{2}+2 b_{n}\left(1-b_{n}\right)\left\|x_{n}-u_{n}\right\|^{2}\left(1-A\left(\left\|x_{n}-u_{n}\right\|\right)\right) \\
& +2 b_{n}\left(1-b_{n}\right) \operatorname{Re}\left\langle S u_{n}-u_{n}, J\left(x_{n}-u_{n}\right)\right\rangle \\
& +D_{3} b_{n} b\left(b_{n}\right)+D_{1}\left\|\delta_{n}\right\|+D_{2}\left\|\delta_{n}\right\| b\left(\left\|\delta_{n}\right\|\right) \\
\leq & \left\|x_{n}-u_{n}\right\|^{2}+D_{4} b_{n}+D_{3} b_{n} b\left(b_{n}\right)+D_{1}\left\|\delta_{n}\right\|+D_{2}\left\|\delta_{n}\right\| b\left(\left\|\delta_{n}\right\|\right)
\end{aligned}
$$

for $n \geq 0$ and some constants $D_{1}>0, D_{2}>0, D_{3}>0$, and $D_{4}>0$. Using Lemma 1.2, (2.1), (2.2), and (2.13), we infer that

$$
\begin{aligned}
\left\|x_{n+1}-u_{n+1}\right\|^{2}= & \left\|\left(1-a_{n}\right)\left(x_{n}-u_{n}\right)+a_{n}\left(S y_{n}-S u_{n}\right)\right\|^{2} \\
\leq & \left(1-a_{n}\right)^{2}\left\|x_{n}-u_{n}\right\|^{2}+2 a_{n}\left(1-a_{n}\right) \operatorname{Re}\left\langle S y_{n}-S u_{n}, J\left(x_{n}-u_{n}\right)\right\rangle \\
& +\max \left\{\left(1-a_{n}\right)\left\|x_{n}-u_{n}\right\|, 1\right\} a_{n}\left\|S y_{n}-S u_{n}\right\| b\left(a_{n}\left\|S y_{n}-S u_{n}\right\|\right) \\
\leq & \left(1-a_{n}\right)^{2}\left\|x_{n}-u_{n}\right\|^{2}+2 a_{n}\left(1-a_{n}\right)\left\|y_{n}-u_{n}\right\|^{2}\left(1-A\left(\left\|y_{n}-u_{n}\right\|\right)\right) \\
& +2 a_{n}\left(1-a_{n}\right) \operatorname{Re}\left\langle S y_{n}-S u_{n}, J\left(x_{n}-u_{n}\right)-J\left(y_{n}-u_{n}\right)\right\rangle+D_{5} a_{n} b\left(a_{n}\right) \\
\leq & \left(1-a_{n}\right)^{2}\left\|x_{n}-u_{n}\right\|^{2}+2 a_{n}\left(1-a_{n}\right)\left(1-A\left(\left\|y_{n}-u_{n}\right\|\right)\right) \\
& \times\left[\left\|x_{n}-u_{n}\right\|^{2}+D_{4} b_{n}+D_{3} b_{n} b\left(b_{n}\right)+D_{1}\left\|\delta_{n}\right\|+D_{2}\left\|\delta_{n}\right\| b\left(\left\|\delta_{n}\right\|\right)\right] \\
& +2 a_{n}\left\|S y_{n}-S u_{n}\right\|\left\|J\left(x_{n}-u_{n}\right)-J\left(y_{n}-u_{n}\right)\right\|+D_{5} a_{n} b\left(a_{n}\right) \\
\leq & \left\|x_{n}-u_{n}\right\|^{2}+\left[d_{n}-A\left(\left\|y_{n}-u_{n}\right\|\right)\left\|x_{n}-u_{n}\right\|^{2}\right] a_{n}
\end{aligned}
$$

for $n \geq 0$ and some constants $D_{5}>0, D>0$, where

$$
d_{n}=D\left[b_{n}+b_{n} b\left(b_{n}\right)+\left\|\delta_{n}\right\|+\left\|\delta_{n}\right\| b\left(\left\|\delta_{n}\right\|\right)+b\left(a_{n}\right)+\left\|J\left(x_{n}-u_{n}\right)-J\left(y_{n}-u_{n}\right)\right\|\right] .
$$

In light of (2.1), (2.4), (2.7)-(2.12), we know that

$$
\left\|x_{n}-u_{n}-y_{n}+u_{n}\right\| \leq b_{n}|| S x_{n}-x_{n}\|+\| \delta_{n} \| \longrightarrow 0 \text { as } n \longrightarrow \infty .
$$


6 The equivalence of Mann and Ishikawa iteration methods

From the continuity of the function $b,(2.4)$ and (2.16), we deduce that

$$
d_{n} \longrightarrow 0 \quad \text { as } n \longrightarrow \infty
$$

Put $\inf \left\{A\left(\left\|y_{n}-u_{n}\right\|\right): n \geq 0\right\}=t$ and $\inf \left\{A\left(\left\|x_{n}-u_{n}\right\|\right): n \geq 0\right\}=r$. Suppose that $r t>0$. From (2.17) we conclude immediately that there exists an integer $m$ such that $d_{n}<1 / 2 r^{2} t$, for all $n \geq m$. By virtue of (2.14) and (2.17), we get that for each $n \geq m$,

$$
\left\|x_{n+1}-u_{n+1}\right\|^{2} \leq\left\|x_{n}-u_{n}\right\|^{2}+\left(d_{n}-r^{2} t\right) a_{n} \leq\left\|x_{n}-u_{n}\right\|^{2}-\frac{1}{2} r^{2} t a_{n},
$$

which implies that

$$
\frac{1}{2} r^{2} t \sum_{k=m}^{\infty} a_{k} \leq\left\|x_{m}-u_{m}\right\|^{2}<\infty,
$$

which contradicts with (2.3). Hence $r t=0$. Without loss of generality we may assume that $t=0$. Clearly there exists a subsequence $\left\{\left\|y_{n_{i}}-u_{n_{i}}\right\|\right\}_{i \geq 0}$ of $\left\{\left\|y_{n}-u_{n}\right\|\right\}_{n \geq 0}$ satisfying $\lim _{i \rightarrow \infty}\left\|y_{n_{i}}-u_{n_{i}}\right\|=0$. In view of (2.1), (2.4), (2.7)-(2.11), we infer that

$$
\left\|x_{n_{i}}-u_{n_{i}}\right\| \leq\left\|y_{n_{i}}-u_{n_{i}}\right\|+b_{n_{i}}|| S x_{n_{i}}-x_{n_{i}}\|+\| \delta_{n_{i}} \| \longrightarrow 0 \quad \text { as } i \longrightarrow \infty .
$$

That is, $\lim _{i \rightarrow \infty}\left\|x_{n_{i}}-u_{n_{i}}\right\|=0$. Using (2.3), (2.4), and (2.14), we conclude easily that for given $\varepsilon>0$ there exists an integer $k$ satisfying

$$
\begin{gathered}
\left\|x_{n_{k}}-u_{n_{k}}\right\|<\frac{\varepsilon}{\sqrt{2}}, \quad d_{n}<\frac{\phi(3 /(8 \sqrt{2}) \varepsilon)}{1+2 L+\phi(2 L)} \frac{9 \varepsilon^{2}}{32}, \\
\max \left\{a_{n}, b_{n}\right\}<\frac{\varepsilon}{8 \sqrt{2} M}, \quad\left\|\delta_{n}\right\|<\frac{\varepsilon}{8 \sqrt{2}}
\end{gathered}
$$

for any $n \geq n_{k}$. Next we prove by induction that for any $i \geq 0$

$$
\left\|x_{n_{k}+i}-u_{n_{k}+i}\right\|<\frac{\varepsilon}{\sqrt{2}} .
$$

In fact, (2.21) implies that (2.22) holds for $i=0$. Suppose that (2.22) holds for some $i>0$. If $\left\|x_{n_{k}+i+1}-u_{n_{k}+i+1}\right\| \geq \varepsilon / \sqrt{2}$, then

$$
\begin{gathered}
\left\|x_{n_{k}+i}-u_{n_{k}+i}\right\| \geq\left\|x_{n_{k}+i+1}-u_{n_{k}+i+1}\right\|-a_{n_{k}+i}|| S y_{n_{k}+i}-S u_{n_{k}+i} \|>\frac{3 \varepsilon}{4 \sqrt{2}}, \\
\left\|y_{n_{k}+i}-u_{n_{k}+i}\right\| \geq\left\|x_{n_{k}+i}-u_{n_{k}+i}\right\|-b_{n_{k}+i}|| S x_{n_{k}+i}-x_{n_{k}+i}\|-\| \delta_{n_{k}+i} \|>\frac{3 \varepsilon}{8 \sqrt{2}} .
\end{gathered}
$$

In view of (2.14), we have

$$
\begin{aligned}
\frac{\varepsilon^{2}}{2} & \leq\left\|x_{n_{k}+i+1}-u_{n_{k}+i+1}\right\|^{2} \\
& \leq\left\|x_{n_{k}+i}-u_{n_{k}+i}\right\|^{2}+\left[d_{n_{k}+i}-A\left(\left\|y_{n_{k}+i}-u_{n_{k}+i}\right\|\right)\left\|x_{n_{k}+i}-u_{n_{k}+i}\right\|^{2}\right] a_{n_{k}+i} \\
& <\frac{\varepsilon^{2}}{2}+\left[d_{n_{k}+i}-\frac{\phi(3 /(8 \sqrt{2}) \varepsilon)}{1+2 L+\phi(2 L)} \frac{9 \varepsilon^{2}}{32}\right] a_{n_{k}+i}<\frac{\varepsilon^{2}}{2}
\end{aligned}
$$


which is impossible. Therefore (2.22) holds for any integer $i \geq 0$. It follows from (2.22) that $\left\|x_{n}-u_{n}\right\| \rightarrow 0$ as $n \rightarrow \infty$. Consequently,

$$
\left\|x_{n}-q\right\| \leq\left\|x_{n}-u_{n}\right\|+\left\|u_{n}-q\right\| \longrightarrow 0 \quad \text { as } n \longrightarrow \infty \text {. }
$$

Hence the Ishikawa iteration sequence with errors $\left\{x_{n}\right\}_{n \geq 0}$ converges strongly to the unique solution of $T x=f$. This completes the proof.

Remark 2.2. (i) If the equation $T x=f$ possesses a solution, then Theorem 2.1 holds without the demicontinuity of $T$.

(ii) If the continuity or Lipschitz continuity of the operator $T$ and the boundedness of the operator $(1-T)$ in $[5,14,20]$ are replaced by the more general demicontinuity and condition (2.5), respectively, then Theorem 2.1 ensures that [5, Theorems 1 and 2], [14, Theorem 1 and Corollary 2], and [20, Theorem 1 and Corollary 1], respectively, are pairwise equivalent.

(iii) Theorem 2.1 extends [18, Corollary 3.1] from the Mann iteration method and the Ishikawa iteration method to the more general Mann iteration method with errors and Ishikawa iteration method with errors, and from the class of strongly accretive operators to the more general class of $\phi$-strongly accretive operators.

The following example shows that Theorem 2.1 generalizes indeed [5, Theorems 1 and 2], [14, Theorem 1 and Corollary 2], [18, Corollary 3.1], [20, Theorem 1 and Corollary $1]$.

Example 2.3. Let $X=(-\infty, \infty)$ with the usual norm $|\cdot|$ and $a_{n}=b_{n}=\delta_{n}=1 /(n+1)$, $\sigma_{n}=1 /(n+1)^{2}, n \geq 0$. Define $T: X \rightarrow X$ and $\phi:[0, \infty) \rightarrow[0, \infty)$ by

$$
\begin{gathered}
T x= \begin{cases}\frac{x^{2}}{3(1+x)} & \text { for } x \in[0, \infty), \\
\frac{1}{3} x-\sqrt{-x} & \text { for } x \in[-1,0), \\
\frac{1}{3} x-1 & \text { for } x \in(-\infty,-1),\end{cases} \\
\phi(t)=\frac{t^{2}}{3(1+t)} \quad \text { for } t \in[0, \infty),
\end{gathered}
$$

respectively. It is easy to see that $T$ is continuous and for any $u_{0}=x_{0}>0,\left\{T x_{n}\right\}_{n \geq 0}$ and $\left\{T y_{n}\right\}_{n \geq 0}$ are bounded, where $\left\{x_{n}\right\}_{n \geq 0}$ and $\left\{y_{n}\right\}_{n \geq 0}$ are as in (2.1). In order to prove that $T$ is $\phi$-strongly accretive, for any $x, y \in X$ with $x \geq y$, we consider the following cases.

Case 1. Suppose that $x, y \in[0, \infty)$. It follows that

$$
\begin{aligned}
& \langle T x-T y, x-y\rangle-\phi(|x-y|)|x-y| \\
& \quad=\frac{1}{3}(x-y)^{2} \frac{y(2+x)}{(1+x)(1+y)(1+x-y)} \geq 0 .
\end{aligned}
$$


8 The equivalence of Mann and Ishikawa iteration methods

Case 2. Suppose that $x, y \in[-1,0)$. It is easy to verify that

$$
\begin{aligned}
& \langle T x-T y, x-y\rangle-\phi(|x-y|)|x-y| \\
& \quad=\frac{1}{3}(x-y)^{2}\left(1-\frac{x-y}{1+x-y}\right)-(\sqrt{-x}-\sqrt{-y})(x-y) \geq 0 .
\end{aligned}
$$

Case 3. Suppose that $x, y \in(-\infty,-1)$. Then we have

$$
\langle T x-T y, x-y\rangle-\phi(|x-y|)|x-y|=\frac{1}{3}(x-y)^{2} \frac{1}{1+x-y} \geq 0 .
$$

Case 4. Suppose that $x \in[0, \infty)$ and $y \in[-1,0)$. It follows that

$$
\begin{aligned}
& \langle T x-T y, x-y\rangle-\phi(|x-y|)|x-y| \\
& \quad=\frac{1}{3}(x-y) \frac{-y}{(1+x)(1+x-y)}+\sqrt{-y}(x-y) \geq 0 .
\end{aligned}
$$

Case 5. Suppose that $x \in[0, \infty)$ and $y \in(-\infty,-1)$. It is easy to see that

$$
\begin{aligned}
& \langle T x-T y, x-y\rangle-\phi(|x-y|)|x-y| \\
& \quad=\frac{1}{3}(x-y) \frac{-y}{(1+x)(1+x-y)}+(x-y) \geq 0 .
\end{aligned}
$$

Case 6. Suppose that $x \in[-1,0)$ and $y \in(-\infty,-1)$. It is easy to verify that

$$
\begin{aligned}
& \langle T x-T y, x-y\rangle-\phi(|x-y|)|x-y| \\
& \quad=\frac{1}{3}(x-y)^{2} \frac{1}{1+x-y}+(1-\sqrt{-x})(x-y) \geq 0 .
\end{aligned}
$$

Therefore, $T$ is $\phi$-strongly accretive. Consequently, Theorem 2.1 ensures the equivalence of the Mann iteration method with errors and the Ishikawa iteration method with errors for $\phi$-strongly accretive operator $T$ in $X$. But the results in $[5,14,18,20]$ are not applicable since $T$ is neither strongly accretive nor Lipschitz. In fact, for any given $\varepsilon \in(0,1)$, there exist $\left.\left(x_{\varepsilon}, y_{\varepsilon}\right)=\varepsilon /(1-\varepsilon), 0\right) \in X \times X$ such that

$$
\left\langle T x_{\varepsilon}-T y_{\varepsilon}, x_{\varepsilon}-y_{\varepsilon}\right\rangle-\varepsilon\left|x_{\varepsilon}-y_{\varepsilon}\right|^{2}=\frac{x_{\varepsilon}^{3}}{3\left(1+x_{\varepsilon}\right)}-\varepsilon x_{\varepsilon}^{2}=-\frac{2 \varepsilon}{3} x_{\varepsilon}^{2}<0,
$$

which yields that $T$ is not strongly accretive. On the other hand,

$$
\lim _{x \rightarrow 0^{-}} \frac{T x-T 0}{x-0}=\lim _{x \rightarrow 0^{-}}\left(\frac{1}{3}-\frac{\sqrt{-x}}{x}\right)=+\infty
$$

that is, $T$ is not Lipschitz.

Next we establish the equivalence between the Mann iteration method with errors and the Ishikawa iteration methods with errors for demicontinuous accretive operators in uniformly smooth Banach spaces. 
Theorem 2.4. Let $X$ be a uniformly smooth Banach space and $T: X \rightarrow X$ a demicontinuous accretive operator. For a given $f \in X$, define $S: X \rightarrow X$ by $S x=f-T x$ for $x \in X$. Assume that $\left\{\sigma_{n}\right\}_{n \geq 0},\left\{\delta_{n}\right\}_{n \geq 0},\left\{a_{n}\right\}_{n \geq 0},\left\{b_{n}\right\}_{n \geq 0},\left\{x_{n}\right\}_{n \geq 0},\left\{y_{n}\right\}_{n \geq 0}$, and $\left\{u_{n}\right\}_{n \geq 0}$ are as in Theorem 2.1 satisfying (2.1)-(2.4). Suppose that either the sequences $\left\{x_{n}+T x_{n}\right\}_{n \geq 0}$ and $\left\{y_{n}+T y_{n}\right\}_{n \geq 0}$ or the sequences $\left\{T x_{n}\right\}_{n \geq 0}$ and $\left\{T y_{n}\right\}_{n \geq 0}$ are bounded. Then, for $u_{0}=x_{0} \in$ $X$, the following assertions are equivalent:

(iii) the Mann iteration sequence with errors $\left\{u_{n}\right\}_{n \geq 0}$ converges strongly to the unique solution of the equation $x+T x=f$;

(iv) the Ishikawa iteration sequence with errors $\left\{x_{n}\right\}_{n \geq 0}$ converges strongly to the unique solution of the equation $x+T x=f$.

Proof. Put $A=I+T$. Clearly, $S x=f+x-A x$ for $x \in X$. It is easy to see that $A: X \rightarrow X$ is demicontinuous $\phi$-strongly accretive operator with $\phi(t)=1 / 2 t$, for all $t>0$. It follows from Lemma 1.1 that $A x=f$ has a unique solution for a given $f \in X$. The sequences $\left\{A x_{n}\right\}_{n \geq 0}$ and $\left\{A y_{n}\right\}_{n \geq 0}$ or the sequences $\left\{x_{n}-A x_{n}\right\}_{n \geq 0}$ and $\left\{y_{n}-A y_{n}\right\}_{n \geq 0}$ are bounded. Hence Theorem 2.4 follows from Theorem 2.1. This completes the proof.

Remark 2.5. Theorem 2.4 generalizes [18, Corollary 3.2].

Theorem 2.6. Let $K$ be a nonempty closed convex subset of a uniformly smooth Banach space $X$ and $T: K \rightarrow K$ a $\phi$-strongly pseudocontractive operator. Suppose that the Ishikawa iteration sequence $\left\{x_{n}\right\}_{n \geq 0}$ is defined by $x_{0} \in X$,

$$
\begin{gathered}
y_{n}=\left(1-b_{n}\right) x_{n}+b_{n} T x_{n}, \\
x_{n+1}=\left(1-a_{n}\right) x_{n}+a_{n} T y_{n}, \quad n \geq 0,
\end{gathered}
$$

and the Mann iteration sequence $\left\{u_{n}\right\}_{n \geq 0}$ by $u_{0} \in X$,

$$
u_{n+1}=\left(1-a_{n}\right) u_{n}+a_{n} T u_{n}, \quad n \geq 0,
$$

where $\left\{a_{n}\right\}_{n \geq 0}$ and $\left\{b_{n}\right\}_{n \geq 0}$ are sequences in $[0,1]$ satisfying

$$
\sum_{n=0}^{\infty} a_{n}=\infty, \quad \lim _{n \rightarrow \infty} a_{n}=\lim _{n \rightarrow \infty} b_{n}=0 .
$$

Assume that $F(T)=\{x: T x=x \in K\} \neq \varnothing$ and

$$
\text { the sequences }\left\{T x_{n}\right\}_{n \geq 0} \text { and }\left\{T y_{n}\right\}_{n \geq 0} \text { are bounded. }
$$

Then, for $u_{0}=x_{0}$, the following assertions are equivalent:

(v) the Mann iteration sequence $\left\{u_{n}\right\}_{n \geq 0}$ converges strongly to the fixed point of T;

(vi) the Ishikawa iteration sequence $\left\{x_{n}\right\}_{n \geq 0}$ converges strongly to the fixed point of $T$. 
Proof. Since $T$ is $\phi$-strongly pseudocontractive and $F(T) \neq \varnothing$, it follows that $T$ has a unique fixed point $q \in K$ and

$$
\langle(I-T) x-(I-T) y, J(x-y)\rangle \geq \phi(\|x-y\|)\|x-y\| \quad \forall x, y \in K .
$$

The rest of the proof is identical with the proof of Theorem 2.1. This completes the proof.

Remark 2.7. If the continuity or Lipschitz continuity of the operator $T$ and the boundedness of the subset $K[1,3-7]$ are replaced by $F(T) \neq \varnothing$ and condition (2.39), respectively, then Theorem 2.6 reveals [1, Theorems 3.2 and 4.1], [3, Theorems 3.3 and 4.1], [4, Theorems 1 and 2], [5, Theorems 3 and 4], [6, the Theorem and Corollary], and [7, Corollaries 1 and 4], respectively, are pairwise equivalent.

Remark 2.8. Theorem 2.6 extends [18, Theorem 2.1] from the class of strongly psuedocontractive operators to larger class of $\phi$-strongly pseudocontractive operators.

The example below demonstrates that Theorem 2.6 generalizes substantially [ 1 , Theorems 3.2 and 4.1], [3, Theorems 3.3 and 4.1], [4, Theorems 1 and 2], [5, Theorems 3 and 4], [6, the Theorem and Corollary], [7, Corollaries 1 and 4], and [18, Theorem 2.1].

Example 2.9. Let $X=(-\infty, \infty)$ with the usual norm $|\cdot|, K=[-1, \infty]$, and $a_{n}=b_{n}=$ $1 /(n+1), n \geq 0$. Define $T: K \rightarrow K$ and $\phi:[0, \infty] \rightarrow[0, \infty]$ by

$$
\begin{aligned}
& T x= \begin{cases}\frac{x}{1+x} & \text { for } x \in[0,+\infty), \\
\sqrt{-x} & \text { for } x \in[-1,0),\end{cases} \\
& \phi(t)=\frac{t^{2}}{1+t} \quad \text { for } t \in[0, \infty),
\end{aligned}
$$

respectively. It is easy to see that $F(T)=\{0\}$ and the range of $T$ is bounded. In order to show that $T$ is $\phi$-strongly pseudocontractive, for any $x, y \in K$ with $x \geq y$, we consider the following cases

Case 1. Suppose that $x, y \in[0, \infty)$. It follows that

$$
\begin{array}{r}
\langle T x-T y, x-y\rangle-|x-y|^{2}+\phi(|x-y|)|x-y| \\
=-(x-y)^{2} \frac{y(2+x)}{(1+x)(1+y)(1+x-y)} \leq 0 .
\end{array}
$$

Case 2. Suppose that $x, y \in[-1,0)$. Then

$$
\begin{aligned}
\langle T x- & T y, x-y\rangle-|x-y|^{2}+\phi(|x-y|)|x-y| \\
& =(\sqrt{-x}-\sqrt{-y})(x-y)-(x-y)^{2} \frac{1}{1+x-y} \leq 0 .
\end{aligned}
$$


Case 3. Suppose that $x, y \in[0, \infty)$ and $y \in[-1,0)$. It is easy to see that

$$
\begin{aligned}
& \langle T x-T y, x-y\rangle-|x-y|^{2}+\phi(|x-y|)|x-y| \\
& \quad=(x-y) \frac{y}{(1+x)(1+x-y)}-\sqrt{-y}(x-y) \leq 0 .
\end{aligned}
$$

Thus $T$ is $\phi$-strongly pseudocontractive in $K$. Consequently, Theorem 2.6 ensures the equivalence of the Mann iteration method and the Ishikawa iteration method for $\phi$ strongly pseudocontractive operator $T$ in $K$. But the results in $[1,3-7,18]$ are not applicable since the subset $K$ is unbounded and $T$ is not strongly pseudocontractive. In fact, for any $t>1$ there exist $\left(x_{t}, y_{t}\right)=((t-1) / 2,0) \in K \times K$ such that

$$
\left\langle T x_{t}-T y_{t}, x_{t}-y_{t}\right\rangle-\frac{1}{t}\left|x_{t}-y_{t}\right|^{2}=\frac{x_{t}^{2}}{1+x_{t}}-\frac{1}{t} x_{t}^{2}=x_{t}^{2} \frac{t-1}{(t+1) t}>0,
$$

that is, $T$ is not strongly pseudocontractive in $K$.

\section{Acknowledgments}

The authors are grateful to the referee for his valuable comments and suggestions. The first author was supported by the Science Research Foundation of Educational Department of Liaoning Province in 2006 and the second author was financially supported by Changwon National University in 2005.

\section{References}

[1] S. S. Chang, Some problems and results in the study of nonlinear analysis, Nonlinear Analysis. Theory, Methods \& Applications 30 (1997), no. 7, 4197-4208.

[2] S. S. Chang, Y. J. Cho, and J. K. Kim, The equivalence between the convergence of modified Picard, modified Mann, and modified Ishikawa iterations, Mathematical and Computer Modelling 37 (2003), no. 9-10, 985-991.

[3] S. S. Chang, Y. J. Cho, B. S. Lee, J. S. Jung, and S. M. Kang, Iterative approximations of fixed points and solutions for strongly accretive and strongly pseudo-contractive mappings in Banach spaces, Journal of Mathematical Analysis and Applications 224 (1998), no. 1, 149-165.

[4] C. E. Chidume, Approximation of fixed points of strongly pseudo-contractive mappings, Proceedings of the American Mathematical Society 120 (1994), no. 2, 545-551.

[5] _ Iterative solution of nonlinear equations with strongly accretive operators, Journal of Mathematical Analysis and Applications 192 (1995), no. 2, 502-518.

[6] __ Global iteration schemes for strongly pseudo-contractive maps, Proceedings of the American Mathematical Society 126 (1998), no. 9, 2641-2649.

[7] C. E. Chidume and M. O. Osilike, Fixed point iterations for strictly hemi-contractive maps in uniformly smooth Banach spaces, Numerical Functional Analysis and Optimization 15 (1994), no. 7-8, 779-790.

[8] Z. Liu, M. Bounias, and S. M. Kang, Iterative approximation of solutions to nonlinear equations of $\phi$-strongly accretive operators in Banach spaces, The Rocky Mountain Journal of Mathematics 32 (2002), no. 3, 981-997.

[9] Z. Liu and S. M. Kang, Convergence theorems for $\phi$-strongly accretive and $\phi$-hemicontractive operators, Journal of Mathematical Analysis and Applications 253 (2001), no. 1, 35-49. 
12 The equivalence of Mann and Ishikawa iteration methods

[10] Stability of Ishikawa iteration methods with errors for strong pseudocontractions and nonlinear equations involving accretive operators in arbitrary real Banach spaces, Mathematical and Computer Modelling 34 (2001), no. 3-4, 319-330.

[11] Iterative solutions of nonlinear equations with $\phi$-strongly accretive operators in uniformly smooth Banach spaces, Computers \& Mathematics with Applications 45 (2003), no. 4-5, 623634.

[12] Z. Liu, S. M. Kang, and S. H. Shim, Almost stability of the Mann iteration method with errors for strictly hemi-contractive operators in smooth Banach spaces, Journal of the Korean Mathematical Society 40 (2003), no. 1, 29-40.

[13] Z. Liu, Y. G. Xu, and Y. J. Cho, Iterative solution of nonlinear equations with $\phi$-strongly accretive operators, Archiv der Mathematik 77 (2001), no. 6, 508-516.

[14] M. O. Osilike, Iterative solution of nonlinear equations of the $\phi$-strongly accretive type, Journal of Mathematical Analysis and Applications 200 (1996), no. 2, 259-271.

[15] S. Reich, An iterative procedure for constructing zeros of accretive sets in Banach spaces, Nonlinear Analysis 2 (1978), no. 1, 85-92.

[16] B. E. Rhoades and S. M. Soltuz, On the equivalence of Mann and Ishikawa iteration methods, International Journal of Mathematics and Mathematical Sciences 2003 (2003), no. 7, 451-459.

[17] The equivalence between the convergences of Ishikawa and Mann iterations for an asymptotically pseudocontractive map, Journal of Mathematical Analysis and Applications 283 (2003), no. 2, 681-688.

[18] _ The equivalence of Mann iteration and Ishikawa iteration for non-Lipschitzian operators, International Journal of Mathematics and Mathematical Sciences 2003 (2003), no. 42, 26452651.

[19] _ The equivalence between the convergences of Ishikawa and Mann iterations for an asymptotically nonexpansive in the intermediate sense and strongly successively pseudocontractive maps, Journal of Mathematical Analysis and Applications 289 (2004), no. 1, 266-278.

[20] H. Y. Zhou, Iterative solutions of nonlinear equations involving strongly accretive operators without the Lipschitz assumption, Journal of Mathematical Analysis and Applications 213 (1997), no. 1, 296-307.

Zeqing Liu: Department of Mathematics, Liaoning Normal University Dalian, Liaoning 116029, China

E-mail address: zeqingliu@sina.com.cn

Li Wang: Department of Science, Shenyang Institute of Aeronautical Engineering Shenyang, Liaoning 110034, China

E-mail address: liwang211@163.com

Jeong Sheok Ume: Department of Applied Mathematics, Changwon National University, Changwon 641-773, South Korea

E-mail address: jsume@changwon.ac.kr

Shin Min Kang: Department of Mathematics and Research Institute of Natural Science,

Gyeongsang National University, Chinju 660-701, South Korea

E-mail address: smkangx@nongae.gsnu.ac.kr 


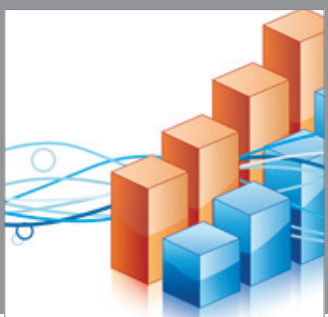

Advances in

Operations Research

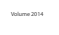

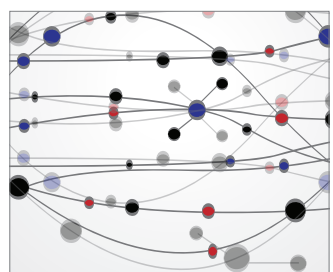

\section{The Scientific} World Journal
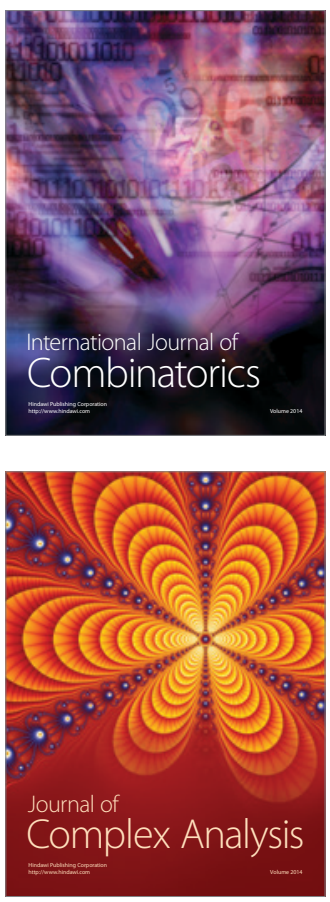

International Journal of

Mathematics and

Mathematical

Sciences
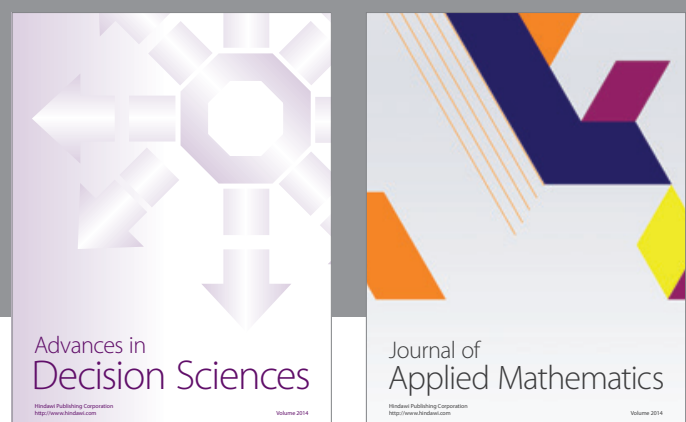

Journal of

Applied Mathematics
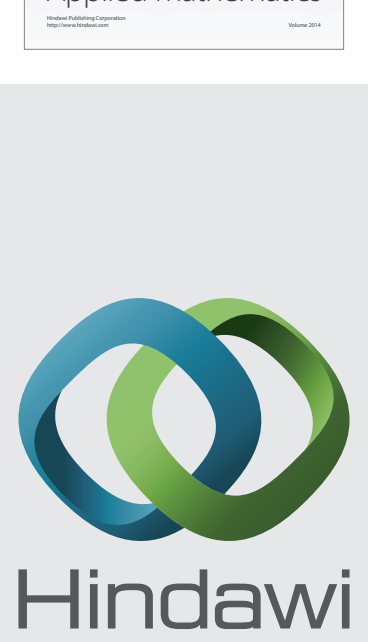

Submit your manuscripts at http://www.hindawi.com
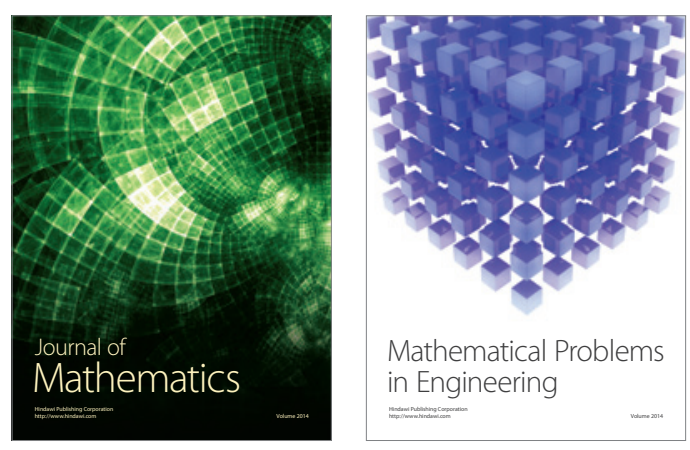

Mathematical Problems in Engineering
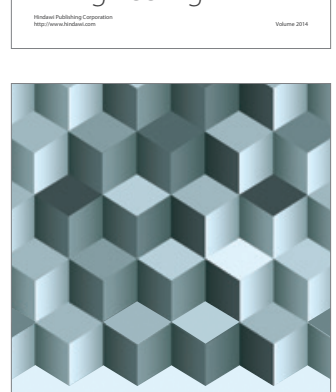

Journal of

Function Spaces
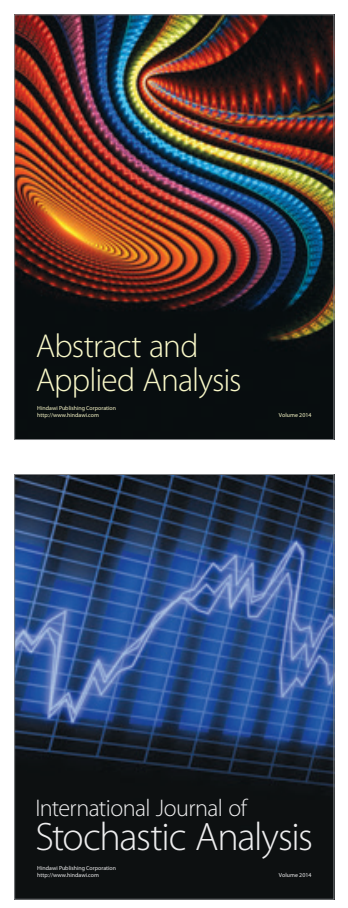

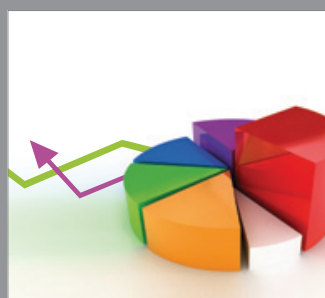

ournal of

Probability and Statistics

Promensencen
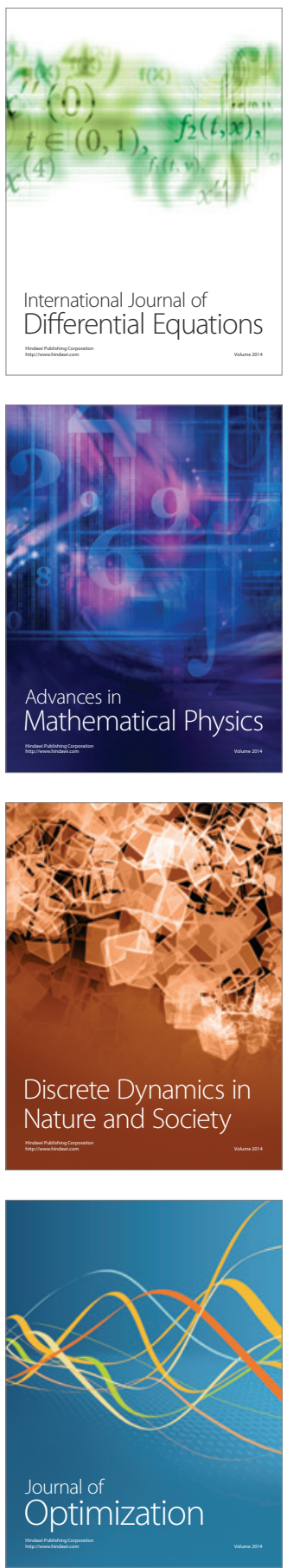